\title{
Article
}

\section{Designing Color Symmetry in Stigmergic Art}

\author{
Hendrik Richter (1)
}

check for updates

Citation: Richter, H. Designing Color Symmetry in Stigmergic Art. Mathematics 2021, 9, 1882. https:// doi.org/10.3390/math9161882

Academic Editor: Dumitru Baleanu

Received: 5 July 2021

Accepted: 5 August 2021

Published: 7 August 2021

Publisher's Note: MDPI stays neutral with regard to jurisdictional claims in published maps and institutional affiliations.
Faculty of Engineering, HTWK Leipzig University of Applied Science, 04277 Leipzig, Germany; hendrik.richter@htwk-leipzig.de

\begin{abstract}
Color symmetry is an extension of the symmetry imposed by isometric transformations and indicates that the colors of geometrical objects are assigned according to the symmetry properties of these objects. A color symmetry permutes the coloring of the objects consistently with their symmetry group. We apply this concept to bio-inspired generative art. Therefore, the geometrical objects are interpreted as motifs that may repeat themselves with a symmetry-consistent coloring. The motifs are obtained by design principles from stigmergy. We discuss the design procedure and present visual results.
\end{abstract}

Keywords: generative art; color symmetry; stigmergy

\section{Introduction}

Symmetry is a major organizational principle in different kinds of design [1,2]. The principle is present in living matter formed by evolutionary processes, as biological entities frequently possess a substantial degree of symmetry. The principle equally applies to man-made objects, whether it be in engineering, where symmetry is economical due to the use of identical components or parts, or in art, where playing with symmetry is a way of expressing aesthetic concepts.

Bio-inspired generative art is a field in evolutionary computation dealing with employing algorithmic ideas and templates observed in nature for creating artworks [3-5]. As symmetry is both an important aesthetic concept in art and an organizational principle in biological entities, it follows rather naturally that different forms and meanings of symmetry matter to bio-inspired generative art, see e.g., [6-9] for explicitly addressing the topic. However, thus far in generative art, there is a certain lack of works dealing with symmetric color transformations.

Thus, in this paper, we focus on a special kind of symmetry: color symmetry. We assume that geometrical objects have symmetry properties that can be described by their symmetry group. If the objects can be colored, then color symmetry implies that the colors of the objects are derived consistently from the symmetry group [8,10-14]. A particularly straightforward method of obtaining symmetry properties frequently employed in visual art is to interpret the geometrical objects as motifs, which may repeat themselves. In this case, color symmetry means a symmetry-consistent coloring of each motif and its copies.

An illustrative example from the history of art for exploring the relationships between geometrical objects, motifs and colors is Vasily Kandinsky's Farbstudie-Quadrate mit konzentrischen Ringen (Color study. Squares with concentric rings), (1913) [15]. Figure 1a shows the study, which consists of 12 squares filling a rectangle of three rows with each square containing a central circle surrounded by concentric rings. Some of the central circles are slightly off-center or almost squarish, and all are surrounded by concentric circles of different colors.

The colors are mainly the primary and the secondary colors of the RYB (red-yellowblue) color wheel $[16,17]$; however, there are also various tertiary colors. Sometimes the circles' colors repeat themselves, and other circles traverse a color space. For instance, we have the sequence (from the inside out) red-blue-red-yellow-blue-black in the upper 
square second to the right. The arrangement can be interpreted as 12 iterations (but also as more or less faithful copies) of a motif.

For a strict symmetry we might expect the geometrical form and/or the color of a constant element within the motif to differ each time, or to stay constant all the time; however, this was not Kandinsky's intention. It is rather an exploration of the motifs taken from basic geometrical forms (squares and circles). In addition, we have variations in the form and color within individual copies and, throughout the study, experiments with different hues and brightness. Nevertheless, we may see Kandinsky's color study as anticipating and preceding some elements of the conceptual framework of color symmetry.

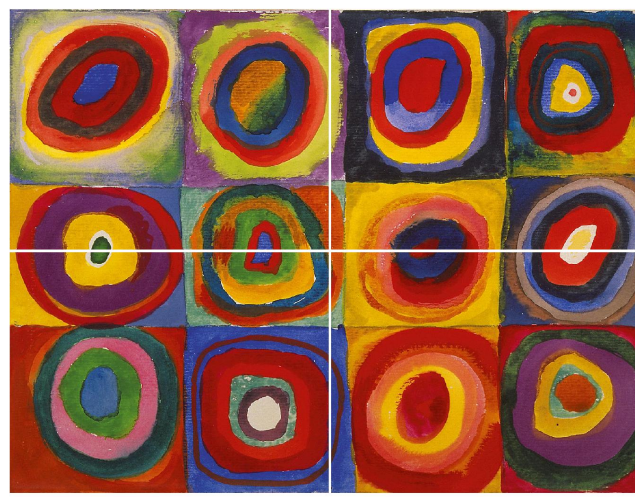

(a)

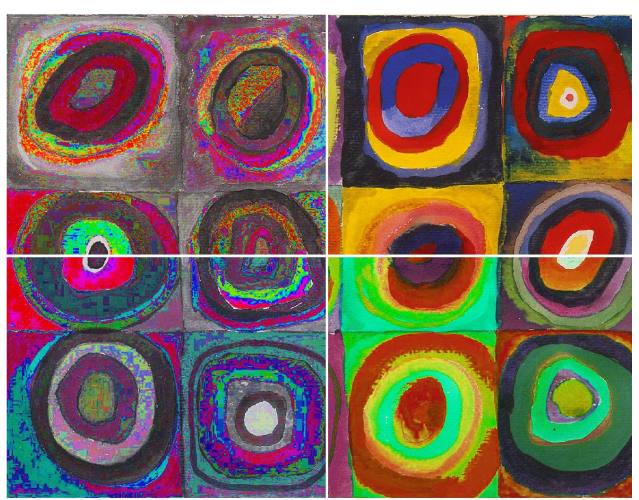

(b)

Figure 1. (a) Vasily Kandinsky (1866-1944): Farbstudie-Quadrate mit konzentrischen Ringen (Color study. Squares with concentric rings), 1913. (b) Color symmetry using a color permutation associated with the symmetry group of the rectangle.

In this paper, the motif generation preceding the actual experiments with color symmetry is performed using algorithms implementing a stigmergic design. Stigmergy is a principle of self-organization in nature, particularly in swarms, see e.g., [7,18-22] for applications in bio-inspired generative art. Similarly to previous works that used Kandinsky's art as an inspiration of generative art $[5,23,24]$, some of the examples of color symmetry shown in this paper adopt some design elements from the color study shown in Figure 1a.

The paper is structured as follows. After these prefatory remarks, Section 2 briefly reviews the meanings and concepts of color, symmetry, and color symmetry in visual art. We also discuss how color symmetry can be imposed by color permutations. We additionally show how color symmetry can be applied to image distortions of pre-existing images. Section 3 recalls the stigmergic generation of geometrical objects and, in particular, shows the motif design used in the paper. Numerical experiments and visual results are given in Section 4, before concluding remarks close the paper with a summary and pointers to future work.

\section{Color, Symmetry, and Color Symmetry}

For describing the significance and meaning of color in art, there is the dictum of the Hungarian-French 20th century painter and graphic designer Victor Vasarely [25]: "Every form is a base for color, every color is the attribute of a form." Put differently, if we see visual art as composed of geometrical objects, then color can be viewed as a property of these objects. If the artistic content of the artwork relates to how and where the geometrical objects are arranged in the image, then different colors allow for distinguishing and contrasting the objects. Suppose there is a point $P_{i}=\left(x_{i}, y_{i}\right)$ from a point set in a two-dimensional $(x, y)$-plane. Then, a color $c_{i}$ is an attribute (or property) of the point $P_{i}$. In other words, a colored point is specified by $P_{i}=\left(x_{i}, y_{i}, c_{i}\right)$. Geometrical objects are built from a set of points. The objects are (monochromatically) colored for all points of the set with the same color. 
A geometrical object may possess symmetry, which implies that the object is invariant under certain transformations $[1,2,26,27]$. The set of transformations under which the geometrical object is symmetric yields the symmetry group $\mathcal{G}$ of the object. On the one hand, we may focus on the symmetry properties of a single geometrical object. For creating or analyzing images, on the other hand, we may consider a geometrical object (which may or may not have symmetry properties itself) as a motif. In an image, such a motif may appear in several copies, and we can study the symmetry properties between these copies.

Suppose we have a circular structure (a wheel) with 12 slots as in Figure 2. Each of the two wheels consists of an inner and an outer circle. Now, let us define a slot to be a motif attributable with a color. Thus, each circle consists of 12 copies of the motif, and we can discuss the symmetry properties. In this discussion, we may assign symmetry to the copies of the motif regardless of the color or taking into account the color. The former implies geometrical symmetry described by isometric transformations, and the latter leads us to color symmetry.

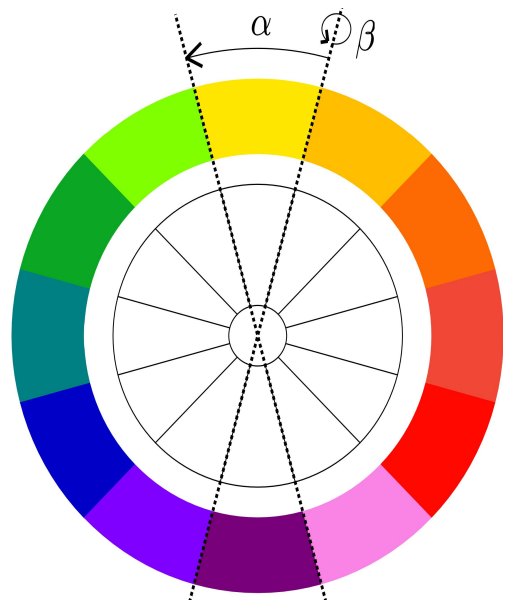

(a)

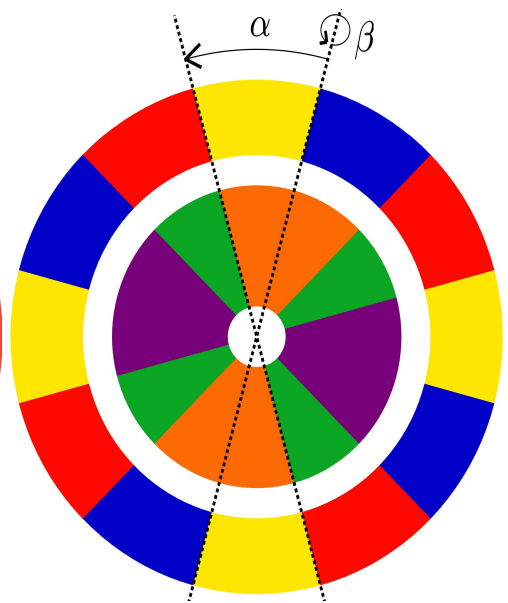

(b)

Figure 2. Symmetry and color symmetry on a color wheel with 12 slots. We denote the slots according to the digits of a clock face. Axes connect slots on the right-hand side of each slot. A rotation $\alpha$ rotates counter-clockwise with $30^{\circ}$, and a reflection $\beta$ reflects in the axis connecting 12 and $6 \mathrm{o}^{\prime}$ clock. (a) The outer circle is the RYB (red-yellow-blue) color wheel. (b) Color symmetry described by the color permutations of Equation (1) (outer circle) and Equation (2) (inner circle).

First, we assume the color of the slots is not taken into account or the color of all the slots is the same, see, for instance, the inner circle of Figure 2a. We denote the slots according to the digits of a clock face with axes connecting slots on the right-hand side of the slot. Then, the symmetry group of the inner circle is the dihedral group $D_{12}$ (or $* 12 \bullet$ in orbifold notation). Let $\alpha$ be a rotation counter-clockwise with $30^{\circ}$ and $\beta$ be a reflection in the axis connecting 12 and $6 o^{\prime}$ clock, see Figure $2 a$ for illustration. We define that symmetries are composed from left to right. For instance, $\beta \alpha$ is first a reflection $\beta$ and then a rotation $\alpha$.

Thus, $\beta \alpha$ is a reflection in the axis connecting 11 and 5 o'clock. Likewise, $\beta \alpha^{2}$ is a reflection in the axis connecting 10 and 4 o'clock, and so on. The symmetry group $\mathcal{G}$ is generated by $\alpha$ and $\beta$ with $e=\alpha^{12}=\beta^{2}, \beta \alpha \beta=\alpha^{11}$. We obtain

$$
\mathcal{G}=\left\{e, \alpha, \alpha^{2}, \ldots, \alpha^{11}, \beta, \beta \alpha, \beta \alpha^{2}, \ldots, \beta \alpha^{11}\right\},
$$

which is of the order 24.

We now add color as an attribute to the slots. The 12 slots can be colored with up to 12 different colors, see the outer circle of Figure 2a and both circles of Figure 2b. The colors of the outer circle of Figure 2a match the RYB (red-yellow-blue) color wheel [16,17], which is also called the standard artistic color wheel. It defines three primary colors: red, yellow, and blue at 4,12, and $8 \mathrm{o}^{\prime}$ clock, respectively, on the circle. Mixing two of these colors each 
gives the three secondary colors: orange (red and yellow), purple (red and blue), and green (yellow and blue) at 2, 6, and 10 o'clock. From these three primary and three secondary colors, we obtain another six tertiary colors by mixing: vermilion (red and orange), amber (orange and yellow), chartreuse (yellow and green), teal (green and blue), violet (blue and purple), and magenta (purple and red). The colors of the circles of Figure $2 \mathrm{~b}$ are also taken from the RYB color wheel.

Now, we consider the color symmetry of the colored slots. An element $g \in \mathcal{G}$ is called a color symmetry if $g$ permutes the coloring of the slots' colors consistently with the symmetry group $\mathcal{G}[11,13,14]$. In other words, $\mathcal{G}$ pre-defines the coloring of each copy of the motif. More specifically, some (or all) elements in $\mathcal{G}$ change colors, while the remaining elements in $\mathcal{G}$ preserve colors. Particularly from an artistic point of view, color symmetry becomes interesting if there is a mix between color-changing and colorpreserving elements of $\mathcal{G}$.

The color symmetry of the outer circle of Figure 2a, which is the RYB circle with 12 colors, is trivial. Of the 24 elements of $\mathcal{G}$, all non-trivial elements change colors, while only the identity $\alpha^{12}=\beta^{2}=e$ preserves colors. As more interesting color symmetries are obtained if the number of copies of the motif exceeds the number of colors, we next consider the color circles with three colors, see Figure 2b, where the outer circle takes the primary colors and the inner circle takes the secondary colors. For such a threefold coloration, the color permutation has order 3 . Recall that the symmetry group $\mathcal{G}$ of the 12-slot circle specifies counter-clockwise rotations about $30^{\circ}$ and reflections along 12 axes. For the outer circle, all reflections $\left\{\beta, \beta \alpha, \beta \alpha^{2}, \ldots, \beta \alpha^{11}\right\}$ change colors (except the trivial $\beta^{2}=e$ ). Rotations $\alpha$ change or preserve the colors according to a color permutation. Using Cauchy's two-line notation, the color permutation associated with the rotation $\alpha$ can be expressed by

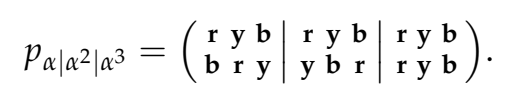

The color permutation implies that the colors are preserved for the rotations $\alpha^{3}, \alpha^{6}$, and $\alpha^{9}$, which are the rotations about $90^{\circ}$.

For the inner circle, almost all rotations $\left\{\alpha, \alpha^{2}, \ldots, \alpha^{11}\right\}$ change color (except the trivial $\alpha^{12}=e$ and $\alpha^{6}$ ). Reflections $\beta$ change or preserve the colors according to the color permutation

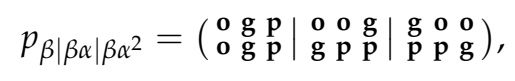

which implies that the reflections $\beta, \beta \alpha^{3}, \beta \alpha^{6}$, and $\beta \alpha^{9}$ preserve colors.

The color symmetry on a wheel with a finite number of slots such as in Figure 2 implies a transitive color permutation, as shown with the examples $p_{\alpha\left|\alpha^{2}\right| \alpha^{3}}$ and $p_{\beta|\beta \alpha| \beta \alpha^{2}}$, see Equations (1) and (2). In other words, for any pair of colors on the wheel, there is an element $g \in \mathcal{G}$ that maps one color of the pair to the other [11]. Possible generalizations could mean that one color is "color-symmetric" to two or more colors, or that an element $g \in \mathcal{G}$ is connected with more than one color mapping, for instance by applying to different sections of the motif differently. Another implication of the color symmetry discussed thus far is that it applies to motifs that are either pre-existing or designed in another way.

We next discuss color symmetry that applies to pre-existing images. Suppose we have a rectangular source images, for instance the Kandinsky study shown in Figure 1a. The symmetry group of the rectangle is the dihedral group $D_{2}$. The rotation $\alpha$ is now counter-clockwise $180^{\circ}$, the reflections $\beta_{h}$ and $\beta_{v}$ are in the horizontal and vertical axis. The symmetry group is of order 4:

$$
\mathcal{G}=\left(e, \alpha, \beta_{h}, \beta_{v}\right)
$$

The reflection axes divide the image into four sections, north-east (ne), north-west (nw), south-east (se), and south-west (sw). Each section can be mapped into any other section by the elements of the symmetry group. The sections are separated in Figure 1a by white lines (which are not present in the original Kandinsky study). We assume that the 
image is composed of $n \times m$ pixels and each pixel $p_{i}$ has a color $c_{i}$ in a HSV color space: $c_{i}=\left(h_{i}, s_{i}, v_{i}\right)$. In the following discussion (and also in the experiments reported later) the focus is on the hue $h_{i}$, while the saturation $s_{i}$ and the value (brightness) $v_{i}$ remain constant; however, of course the same method can be applied to the whole HSV color space.

We now define the color permutation associated with the symmetry group by a map $f\left(h_{i}\right)$, which is a mapping of the hue component of the HSV color space onto itself. Thus, for rectangular source images, a color symmetry can be imposed by a color permutation as follows. We define a reference section, for instance north-east (ne). The colors in this section remain unchanged. The colors of the pixels of the other sections are changed according to the rectangle's symmetry group. For instance, the color permutation associated with the rotation is

$$
p_{\alpha}=\left(\begin{array}{c}
\mathbf{h}_{\mathbf{i}} \\
\mathbf{f}_{\mathbf{1}}\left(\mathbf{h}_{\mathbf{i}}\right)
\end{array}\right),
$$

which implies that the hue $h_{i}$ of all pixels $p_{i}$ in sw is changed from $h_{i}$ to $f_{1}\left(h_{i}\right)$. For the remaining sections $\mathbf{n w}$ and se, we may, likewise, define for the reflections

$$
p_{\beta_{v}}=\left(\begin{array}{c}
\mathbf{h}_{\mathbf{i}} \\
\mathbf{f}_{2}\left(\mathbf{h}_{\mathbf{i}}\right)
\end{array}\right), \quad p_{\beta_{h}}=\left(\begin{array}{c}
\mathbf{h}_{\mathbf{i}} \\
\mathbf{f}_{3}\left(\mathbf{h}_{\mathbf{i}}\right)
\end{array}\right) .
$$

Figure $1 \mathrm{~b}$ shows an example using the Kandinsky study (Figure 1a) as a source image. As color maps, the functions $f_{1}\left(h_{i}\right)=0.45\left|\sin \left(\sqrt{2} \cdot 20 \pi h_{i}\right)\right|+0.55\left|\sin \left(20 \pi h_{i}\right)\right|$, $f_{2}\left(h_{i}\right)=0.5\left(1+\sin \left(40 \pi h_{i}\right)\right)$, and $h_{3}\left(h_{i}\right)=4 h_{i}\left(1-h_{i}\right)$ are used.

Such a design of color symmetry implies that the color permutations according to Equations (4) and (5) most likely still have finite order. Of course, any color described by the hue $h_{i} \in \mathbb{R}$ could be mapped to another color $f\left(h_{i}\right)$. However, most likely, the HSV color space used in computer graphics effectively imposes a finite number of different colors. Nevertheless, the number of different colors might be very large, which opens up a rich variety of color effects.

The visual result in Figure $1 \mathrm{~b}$ shows a substantial variety of color shifts in the sections affected by color symmetry, particularly for the color maps involving trigonometric functions. As the saturation and brightness of the pixels are preserved while the hue is changed, the "color symmetric" sections of the image resembles the original image; however, we also have a kind of alienation effect (or distancing effect) in terms of color.

In Section 4, generative art, which is the result of experiments with color, symmetry, and color symmetry, is presented and discussed. This is done in connection with stigmergic generation algorithms, which are discussed next.

\section{Stigmergy and Generative Art}

Stigmergy is a bio-inspired paradigm of self-organization in swarms. The members of the swarm coordinate themselves by indirect communication, for instance by deposing and collecting visual, olfactory or tactile markers. This paradigm has been used as a computational framework for generating swarm art, see for example [3-5,7,18,19,28,29].

In the experiments with designing color symmetry presented in this paper, we used a model of stigmergic nest construction. The model describes the nest building by the ant species T. albipennis and was proposed by Urbano [22]. The nests are constructed by the ants collecting sand grains from a foraging ground and deposing them to build a closed curved outer boundary wall around the nest. Different colonies of ants may be sensitive to sand with different colors, thus, making it possible to construct polychromatic swarm paintings.

The nest construction modelling T. albipennis behavior builds toroidal walls $[7,22]$. However, it was suggested by Greenfield [19] that the boundary walls could also follow other types of curves, which would make them more interesting than circles from a geometrical point of view. In such an interpretation of boundary walls, different colonies would be sensitive to different types of walls. In the following, this idea is adopted and the stigmergic art discussed In this paper is constructed from the following algorithmic framework. Each nest has a center point $P_{i}=\left(x_{i}, y_{i}\right)$ in a $2 \mathrm{D}$ plane. The nest is further characterized by a boundary wall with radius $r_{i}(\theta)$, which describes the distance to the center point in polar 
coordinates with $\theta=[0,2 k \pi], k=1,2,3, \ldots$ For instance, by setting $k=1$, circles can be constructed by $r_{i}(\theta)=r_{i}=$ const., and $\ell$-leaf roses are obtained for $r_{i}(\theta)=a \sin (\ell \theta)$ and $r_{i}(\theta)=a \cos (\ell \theta)$. Archimedean spirals are described by $r_{i}(\theta)=\gamma+\delta \theta$, with $\gamma, \delta>0$ and $k$ specifying the desired length of the spiral.

The nest's boundary wall is built by ants collecting grain with the nest's boundary type from the foraging ground. An ant has a certain distance from the nest's center point and can either carry a grain or not. If it carries a grain it moves randomly toward the center point and deposes the sand grain with a certain probability within the vicinity of the radius $r_{i}(\theta)$. Using parameters, we can control the speed of nest formation and also the wall thickness. If the ant does not carry a grain, it moves randomly away from the center point and collects a sand grain with a second probability.

Again, we can control, by parameters, the collecting speed and foraging range. The visual effect is that boundary walls are built that have a grain density resembling realizations of a random variable with the center $r_{i}(\theta)$-for instance, a Gaussian distribution with $\mathcal{N}\left(r_{i}(\theta), \sigma\right)$. Contrary to previous works [7,19,22], we assume that the foraging ground is completely emptied in the nest building activity. Therefore, we can describe the $i$-th nest by its center point, its color, and its radius: $N_{i}=\left(x_{i} \cdot y_{i}, c_{i}, r_{i}(\theta)\right)$. We consider these nests as motifs.

\section{Experiments and Visual Results}

In the sections above, a framework was set out for the stigmergic generation of motifs. In addition, the motifs may be the object of experiments designing color symmetries. In the following, we present and discuss artistic experiments generating visual art using this framework.

A first set of experiments takes the nests generated by stigmergic design, colors the grains according to color-symmetric principles, and places them on a black canvas. The examples in Figure 3 are directly inspired by Kandinsky's color study shown in Figure 1a. The images seize the idea of 12 squares filling a rectangle in three rows. The squares contain circles as well as Archimedean spirals and $\ell$-leaf roses. The nests are colored with elements from the RYB color wheel (Figure 2a). The 12 squares fit exactly the whole set of primary, secondary, and tertiary colors of the RYB wheel.

Accordingly, the upper left image (Figure 3a), for instance, has six squares and six inner circles on the left hand side with primary and secondary colors, while the outer circles are the complementary colors (which are exactly opposite on the RYB color wheel). The six remaining squares on the right hand side of the image do the same with the tertiary colors.

Thus, a vertical reflection imposes a color symmetry from primary and secondary colors to tertiary colors. Based on the symmetry group (3), the image is generated by the

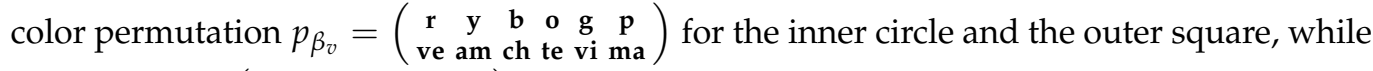

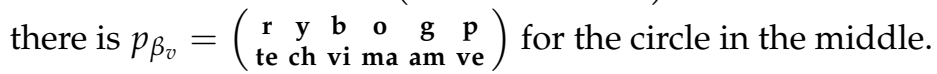

The other images similarly play with squares, circles, Archimedean spirals, and $\ell$-leaf roses and the color symmetries between the RYB colors. Of particular note, in the lower left image, the primary colors are preserved by vertical reflection, while the secondary colors may change. The images in Figure 4 leave behind the rows-and-columns arrangement and place the nests at any prescribed locations on the canvas. Again, isometric symmetry induces color symmetry.

In the upper right image (Figure $4 \mathrm{~b}$ ), the geometrical aspect of symmetry is almost invisible; however, we have color symmetry insofar as any circle with an Archimedean spiral has an color-symmetric counterpart, which is obtained by a rotation about a random center point. The radii of the smallest seven circles in the lower left image (Figure 4d) follow a Fibonacci series with the circles on the left-hand side of the image following a Fibonacci circle curve as proposed by Happersett [30]. The Fibonacci circle curve places subsequent circles according to the Golden Angle $180^{\circ}(3-\sqrt{5})$.

A second set of experiments uses the framework of stigmergic art and color symmetry to generate images based on source images. Modifying the source images with the aim 
of creating visual art that resembles known pictures or paintings but also provides a reinterpretation has also been a topic in bio-inspired generative art. For example, see the modification of source images from the history of art by stochastic hillclimbers, plant propagation algorithms, simulated annealing, or particle swarms [31,32], as well as image transitions by random walks $[5,23,33]$. In the experiments reported here, two well-known painting by Johannes Vermeer were used: Girl with a Pearl Earring (1665) and View of Delft (1661). (Figure 5a,b).

In a first step, the colors in these images were modified using color permutations similar to Equations (4) and (5). In addition to the functions given above, the color maps $f_{4}\left(h_{i}\right)=4 h_{i}\left(h_{i}-1\right)+1, f_{5}=0.15\left(1+\cos \left(40 \pi h_{i}\right)\right)+0.5 h_{i}$, and $f_{k}\left(h_{i}\right)=k h_{i}$ mod 1 were used, see the examples of a triangular pattern and a chess board pattern with 16 elements in Figure 5c,d.

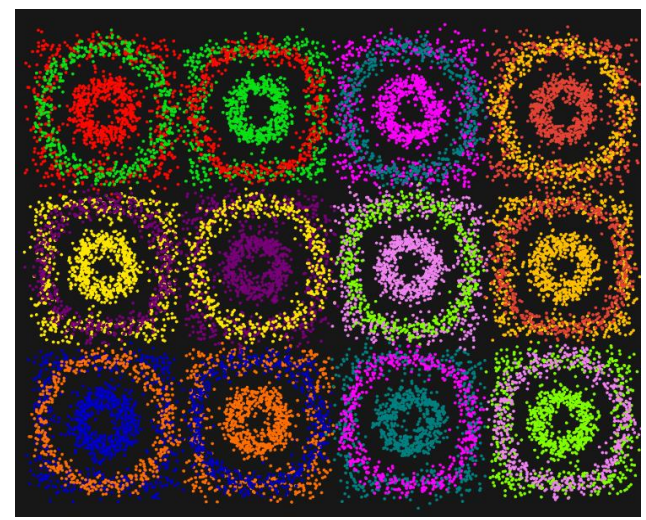

(a)

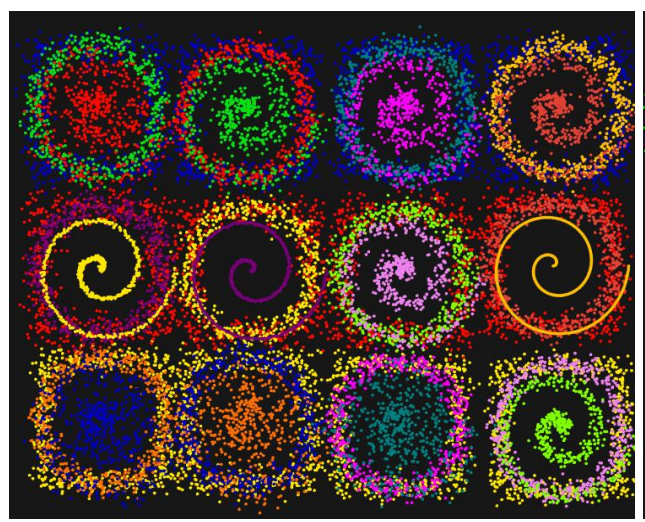

(c)

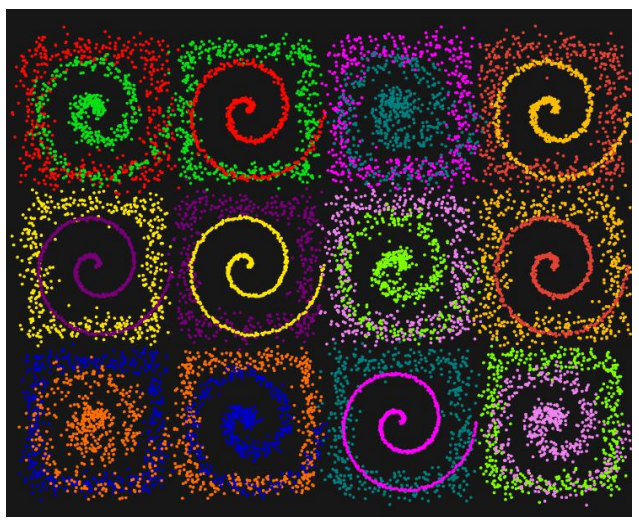

(b)

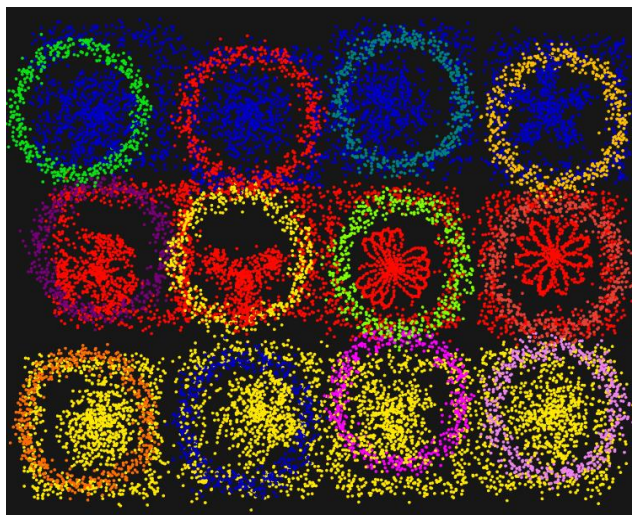

(d)

Figure 3. Four different stigmergic color-symmetric patterns with reminiscence of Kandinsky's color study in Figure 1a. (a) Vertical reflection imposing a color symmetry of circles and squares from primary and secondary colors to tertiary colors. (b) Vertical reflection imposing a color symmetry of Archimedean spirals and squares from primary and secondary colors to tertiary colors. (c) Color reversed version of (b). (d) Vertical reflection imposing a color symmetry of circles and squares which preserve primary colors and change secondary colors to tertiary colors. 


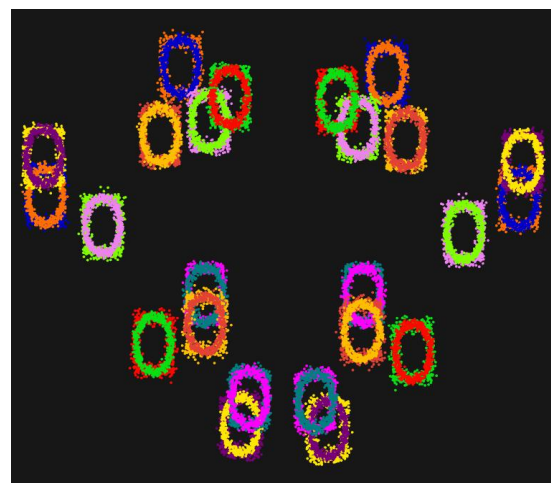

(a)

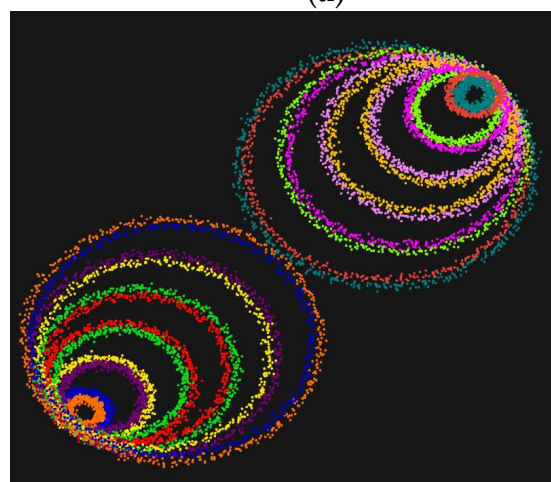

(c)

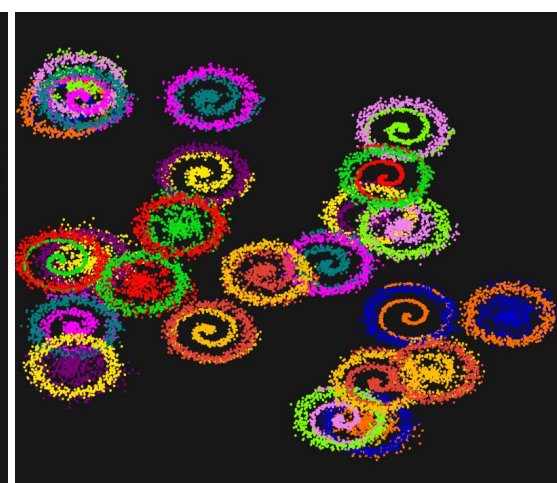

(b)

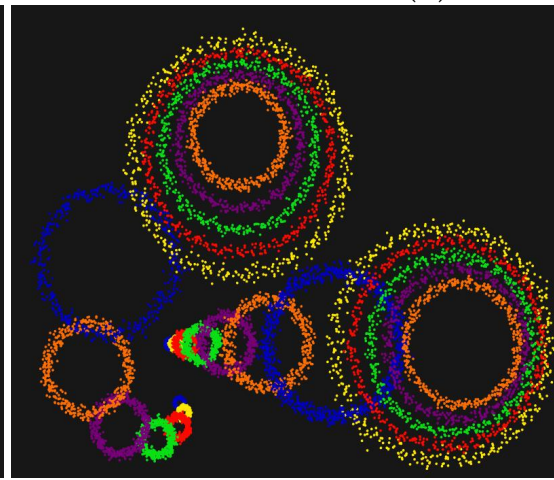

(d)

Figure 4. Four different stigmergic color-symmetric patterns with isometric as well as color symmetry. (a) Vertical reflection (b) Archimedean spirals with color-symmetric counterpart obtained by a rotation about a random center point. (c) Diagonal reflection (d) Circle symmetry following a Fibonacci circle curve.

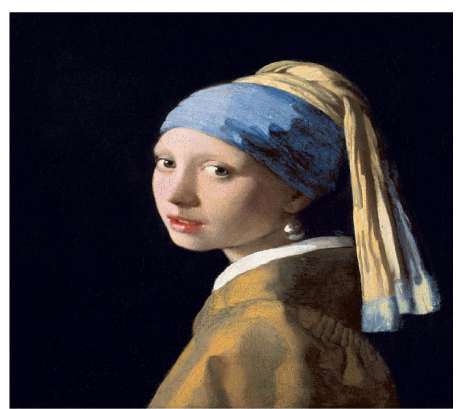

(a)

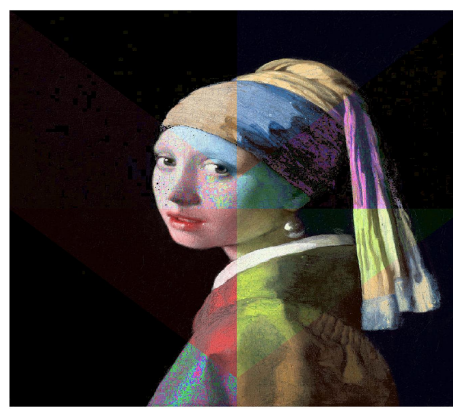

(c)

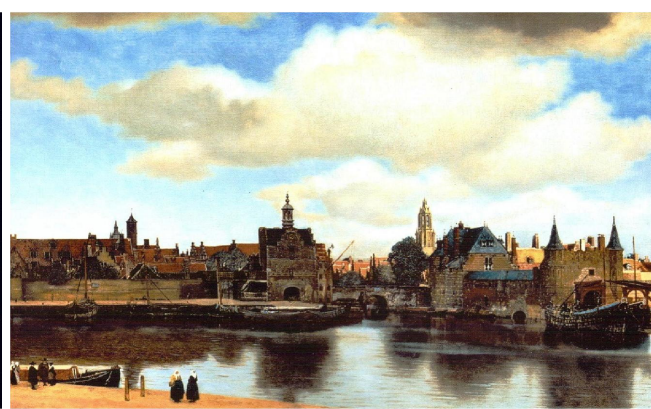

(b)

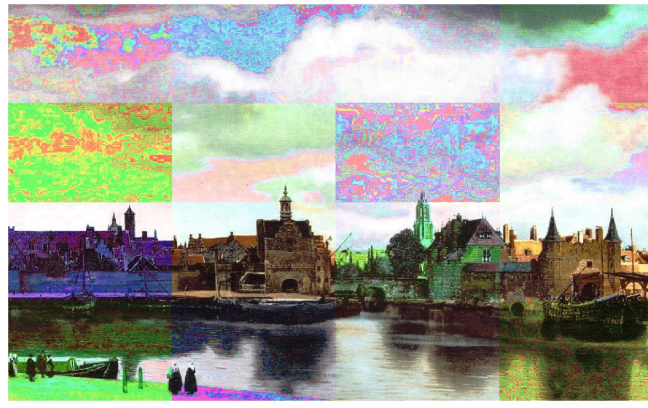

(d)

Figure 5. Johannes Vermeer (1632-1675): (a) Girl with a Pearl Earring, 1665. (b) View of Delft, 1661. The same Vermeer painting with color symmetry imposed by a color permutation. (c) Triangular pattern. (d) Chess board pattern with 16 elements. 
An initial experiment shows color symmetry imposed by triangular section of the rectangular source image, see Figure 6. In the left image, all reflections change colors, while, in the right image, up-down reflection preserves colors. Thus, the images are generated by color permutations as defined by Equation (5). For further examples, see also [9].
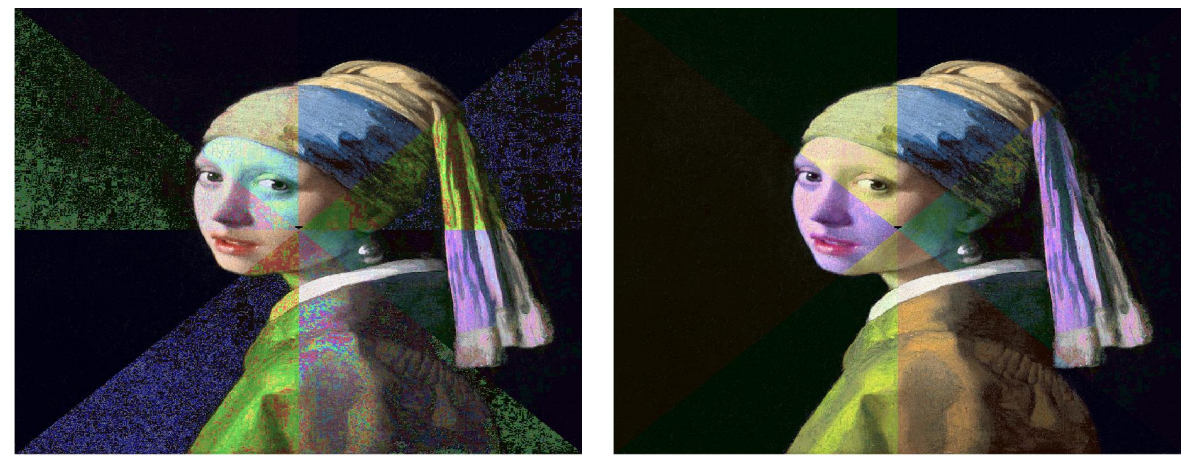

Figure 6. Color symmetry of the full image of Vermeer's Girl with a Pearl Earring, see Figure 5a,c.

Subsequently, patterns of nests are generated where the grains take the colors of the source image while the grains of the symmetric nest patterns take the color-symmetric hues. Figures 7 and 8 show the results, which can be seen as a quotation and homage to Vermeer's famous paintings. Again, the original art works remain recognizable, while, at the same time, an alienation or distancing effect occurs.
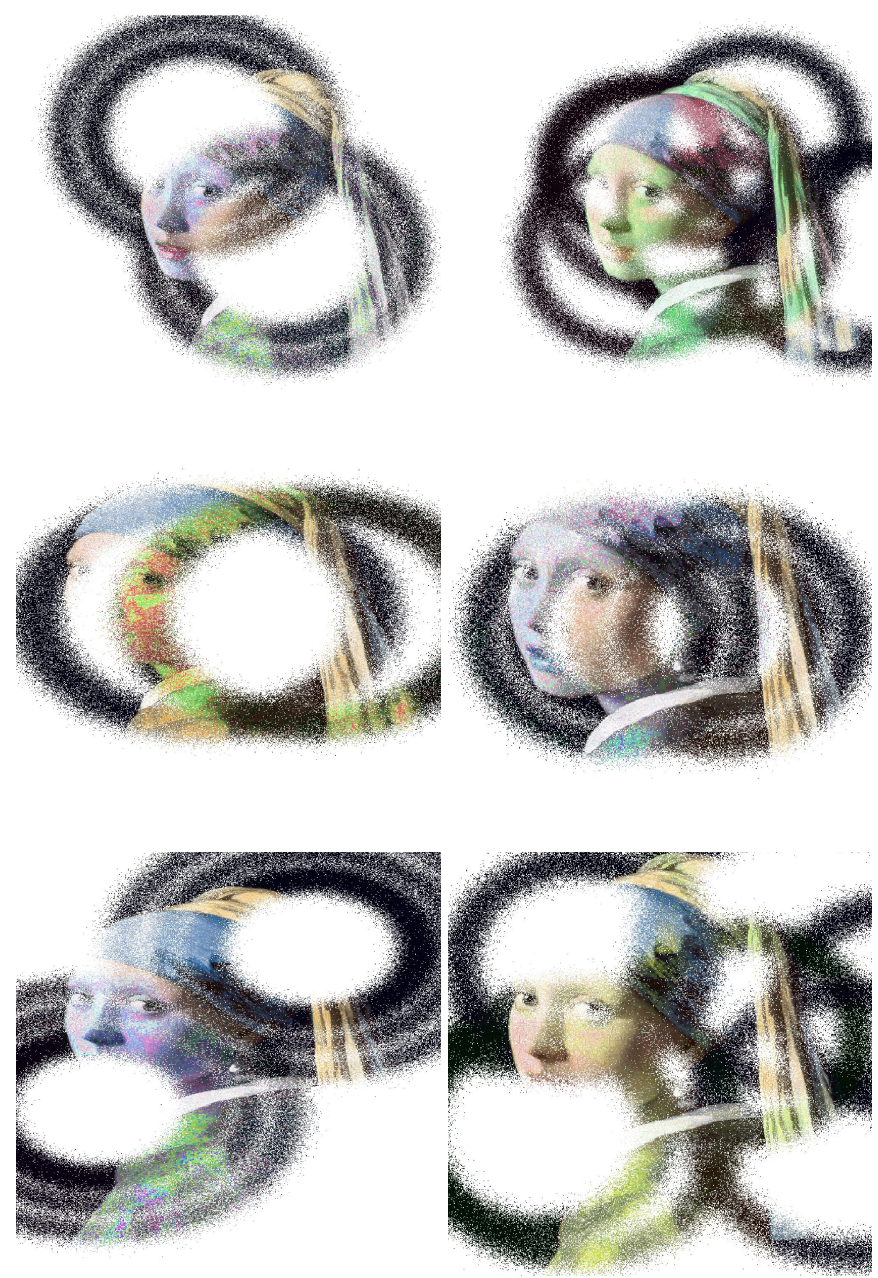

Figure 7. Color symmetric art works, which can be seen as a quotation and homage to Vermeer's Girl with a Pearl Earring. 

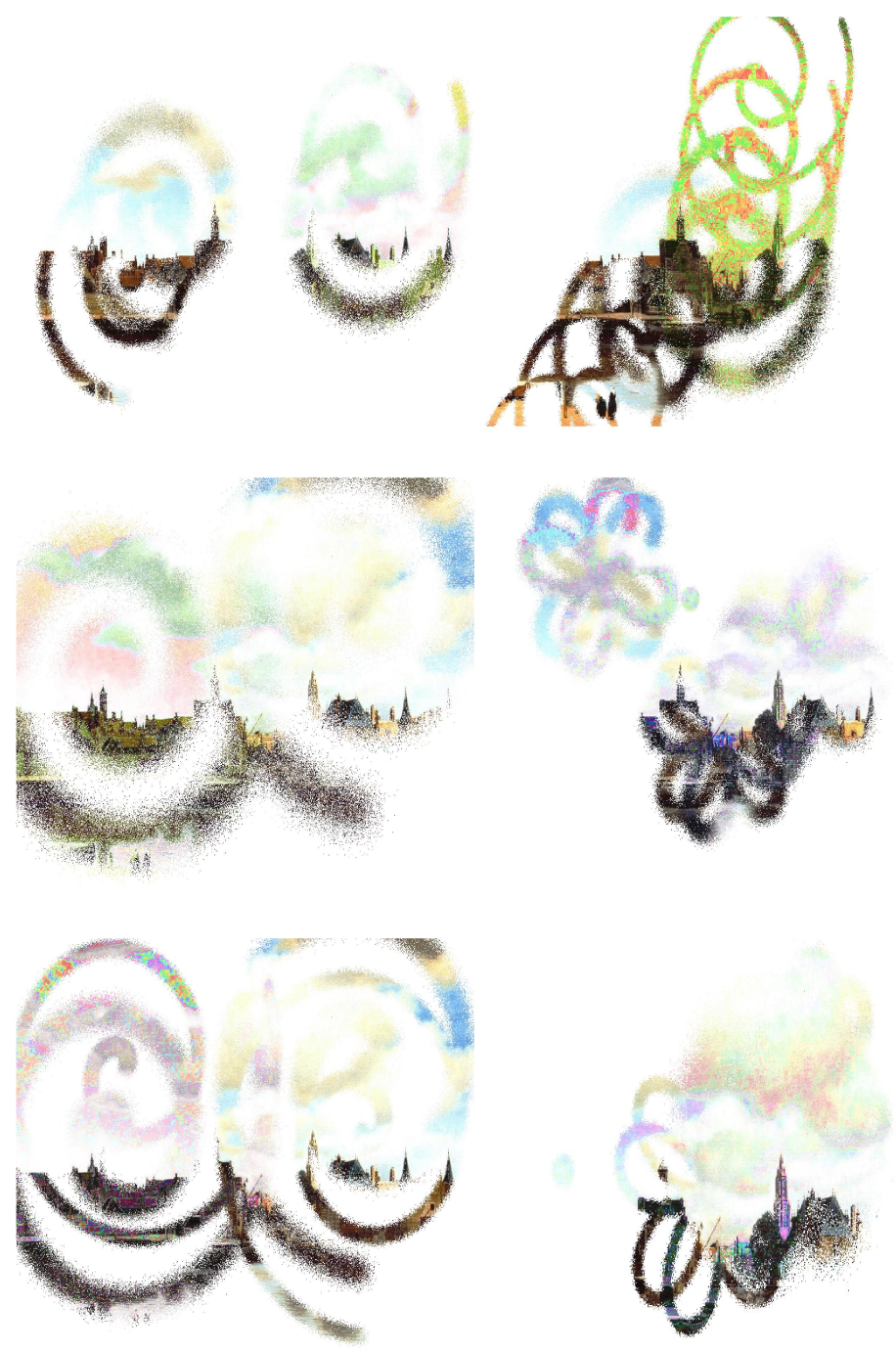

Figure 8. Color symmetric art works quoting Vermeer's View of Delft.

\section{Conclusions}

An important property of symmetry is the repetition of motifs with some features of the motif changing while some other features remain invariant. Take, for instance, a mirror image of a motif, which appears as an almost identical copy but is reversed in the direction perpendicular to the mirror surface. Color symmetry permutes the coloring of objects consistently with their symmetry group. Thus, it implies a symmetry-consistent coloring of the motif and its copies. If a motif adheres to a certain coloring scheme, then the mirror image should change or preserve the colors consistently.

In this paper, experiments with and the visual results of designing such color symmetries were discussed regarding bio-inspired generative art using principles from stigmergy. Therefore, a model of stigmergic nest construction proposed by Urbano [22] (see also Greenfield $[7,19]$ for further usage in generative art) was used to produce geometrical structures. These structures were interpreted as motifs. In the visual results, the stigmergic nest motifs were colored according to algorithmic ideas exploiting symmetry and color symmetry.

The results given in the paper only used a small subset of the design space spanned by the algorithmic framework. Thus, future work could explore the possibilities of the design space to a larger extent. For instance, the focus of color changing maps was on hue $h_{i}$. Therefore, different hue mappings $f\left(h_{i}\right)$ were discussed, while the value (brightness) $v_{i}$ and the saturation $s_{i}$ remained constant. 
As the same method can be used for the whole HSV color space, it would be interesting to simultaneously change these elements of the color space to address different aspects of symmetry at the same time. In addition, it would be interesting to evaluate the results by computational aesthetic measures in order to learn more about how aesthetic feature values are modified by color-symmetric changes. Analogue hardware implementations similar to drawing machines [34] featuring ideas from color symmetry would also be an interesting field of future work.

Funding: This research received no external funding.

Institutional Review Board Statement: Not applicable.

Informed Consent Statement: Not applicable.

Data Availability Statement: Not applicable.

Conflicts of Interest: The authors declare no conflict of interest.

\section{References}

1. Conway, J.H.; Burgiel, H.; Goodman-Strauss, C. The Symmetries of Things; A K Peters: Wellesley, MA, USA, 2008.

2. Weyl, H. Symmetry; Princeton University Press: Princeton, NJ, USA, 1952.

3. Greenfield, G.; Machado, P. Ant- and ant-colony-inspired Alife visual art. Artif. Life 2015, 21, 293-306. [CrossRef] [PubMed]

4. Jacob, C.J.; Hushlak, G.; Boyd, J.E.; Nuytten, P.; Sayles, M.; Pilat, M. Swarmart: Interactive art from swarm intelligence. Leonardo 2007, 40, 248-254. [CrossRef]

5. Neumann, A.; Alexander, B.; Neumann, F. Evolutionary image transition and painting using random walks. Evol. Comput. 2020, 28, 643-675. [CrossRef] [PubMed]

6. Al-Rifaie, M.M.; Ursyn, A.; Zimmer, R.; Javid, M.A.J. On symmetry, aesthetics and quantifying symmetrical complexity. In Computational Intelligence in Music, Sound, Art and Design. EvoMUSART 2017; Correia, J., Ciesielski, V., Liapis, A., Eds.; Springer: Cham, Switzerland, 2017; pp. 17-32.

7. Greenfield, G. Stigmmetry prints from patterns of circles. In Proceedings Bridges 2012: Mathematics, Music, Art, Architecture, Culture; Bosch, R., McKenna, D., Sarhangi, R., Eds.; Tessellations Pub.: Phoenix, AZ, USA, 2012; pp. 291-298.

8. Richter, H. Generating symmetry and symmetry breaking in sand-bubbler patterns. In Proceedings Artificial Life Conference 2020 (ALIFE 2020); Bongard, J., Lovato, J., Hebert-Dufrésne, L., Dasari, R., Soros, L., Eds.; MIT Press: Boston, MA, USA, 2020; pp. 509-517.

9. Richter, H. Wrong colored Vermeer: Color-symmetric image distortion. arXiv 2021, arXiv:2106.15179.

10. Coxeter, H.S.M. Coloured symmetry. In M.C. Escher: Art and Science; Coxeter, H.S.M., Emmer, M., Penrose, R., Teuber, M.L., Eds.; Elsevier: Amsterdam, The Netherlands, 1986; pp. 15-33.

11. Roth, R.L. Color symmetry and group theory. Discret. Math. 1982, 38, 273-296. [CrossRef]

12. Schwarzenberger, R.L.E. Colour symmetry. Bull. Lond. Math. Soc. 1984, 16, 209-240. [CrossRef]

13. Senechal, M. Coloring symmetrical objects symmetrically. Math. Mag. 1983, 56, 3-16. [CrossRef]

14. Senechal, M. Color symmetry. Comp. Math. Appl. 1988, 16, 545-553. [CrossRef]

15. Becks-Malorny, U. Wassily Kandinsky. In The Journey to Abstraction; Taschen: Köln, Germany, 2007; pp. 1866-1944.

16. Itten, J. The Art of Color: The Subjective Experience and Objective Rationale of Color; Van Nostrand Reinhold: New York, NY, USA, 1973.

17. Rhyne, T.M. Applying Color Theory to Digital Media and Visualization; CRC Press: Boca Raton, FL, USA, 2017.

18. Al-Rifaie, M.M.; Bishop, J.M. Swarmic paintings and colour attention. In Evolutionary and Biologically Inspired Music, Sound, Art and Design. EvoMUSART 2013; Machado, P., McDermott, J., Carballal, A., Eds.; Springer: Berlin/Heidelberg, Germany, 2013; pp. 97-108.

19. Greenfield, G. Target curves for pick-up, carry and drop mobile automata. In Proceedings Bridges 2014: Mathematics, Music, Art, Architecture, Culture; Greenfield, G., Sarhangi, R., Eds.; Tessellations Pub.: Phoenix, AZ, USA, 2014; pp. 381-384.

20. Moura, L.; Ramos, V. Swarm paintings-nonhuman art. In Architopia Book, Art, Architecture and Science; Maubant, J., Ed.; Institut d'Art Contemporain: Lyon/Villeurbanne, France, 2002; pp. 5-24.

21. Urbano, P. Playing in the pheromone playground: Experiences in swarm Painting. In EvoWorkshops 2005. LNCS, Vol. 3449; Rothlauf, F., Branke, J., Cagnoni, S., Corne, D.W., Drechsler, R., Jin, Y., Machado, P., Marchiori, E., Romero, J., Smith, G.D., et al., Eds.; Springer: Berlin/Heidelberg, Germany, 2005; pp. 527-532.

22. Urbano, P. The T. albipennis sand painting artists. In Applications of Evolutionary Computation. EvoApplications 2011; Di Chio, C., Ed.; Springer: Berlin/Heidelberg, Germany, 2011; pp. 414-423.

23. Neumann, A.; Alexander, B.; Neumann, F. Evolutionary image transition using random walks. In Computational Intelligence in Music, Sound, Art and Design. EvoMUSART 2017; Correia, J., Ciesielski, V., Liapis, A., Eds.; Springer: Cham, Switzerland, 2017; pp. 230-245.

24. Zhang, K.; Yu, J. Generation of Kandinsky art. Leonardo 2016, 49, 48-54. [CrossRef] 
25. Dresp-Langley, B.; Reeves, A. Color for the perceptual organization of the pictorial plane: Victor Vasarely's legacy to Gestalt psychology. Heliyon 2020, 6, 04375. [CrossRef] [PubMed]

26. Liu, Y.; Hel-Or, H.; Kaplan, C.S.; Van Gool, L. Computational symmetry in computer vision and computer graphics. In Foundations and Trends in Computer Graphics and Vision 5: 1-2; Now Publishers Inc.: Delft, The Netherlands, 2010; pp. 1-195.

27. Martin, G.E. Transformation Geometry: An Introduction to Symmetry; Springer: New York, NY, USA, 1982.

28. Fernes, C.M.; Mora, A.M.; Merelo, J.J.; Rosa, A.C. KANTS: A stigmergic ant algorithm for cluster analysis and swarm art. IEEE Trans. Cybern. 2014, 44, 843-856.

29. Richter, H. Visual art inspired by the collective feeding behavior of sand-bubbler crabs. In Computational Intelligence in Music, Sound, Art and Design. EvoMUSART 2018; Liapis, A., Romero Cardalda, J.J., Ekárt, A., Eds.; Springer: Cham, Switzerland, 2018; pp. 1-17.

30. Happersett, S. Mathematical sequences and artists' books. In Proceedings Bridges 2014: Mathematics, Music, Art, Architecture, Culture; Greenfield, G., Sarhangi, R., Eds.; Tessellations Pub.: Phoenix, AZ, USA, 2014; pp. 385-388.

31. de Andrade, D.; Fachada, N.; Fernes, C.M.; Rosa, A.C. Generative art with swarm landscapes. Entropy 2020, 22, 1284. [CrossRef] [PubMed]

32. Paauw, M.; van den Berg, D. Paintings, polygons and plant propagation. In Computational Intelligence in Music, Sound, Art and Design. EvoMUSART 2019; Springer: Cham, Switzerland, 2019; pp. 84-97.

33. Alexander, B.; Kortman, J.; Neumann, A. Evolution of artistic image variants through feature based diversity optimisation. In Proceedings of the Genetic and Evolutionary Computation Conference, GECCO 2017, Berlin, Germany, 15-19 July 2017; Bosman, P.A.N., Ed.; ACM: New York, NY, USA, 2017; pp. 171-178.

34. Tait, J. Secondary, near chaotic patterns from analogue drawing machines. Mathematics 2019, 7, 86. [CrossRef] 